\title{
Local field enhancement effects for nanostructuring of surfaces
}

\author{
H.-J. MÜNZER*, M. MOSBACHER*†, M. BERTSCH*, J. ZIMMERMANN*, P. LEIDERER* \\ \& J. BONEBERG* \\ *University of Konstanz, Department of Physics, Center of Modern Optics, Fach M676, D-78457 \\ Konstanz, Germany \\ †Johannes-Kepler-University, Applied Physics Institute, Altenbergstr. 69, A-4040 Linz, Austria
}

Key words. Colloids, field enhancement, laser cleaning, Mie scattering, nano-optics, nanostructuring.

\section{Summary}

We report on a method that allows the nanostructuring of surfaces with intense laser pulses. For this purpose isolated polystyrene spheres with diameters in the order of the laser wavelength were deposited on a silicon or glass surface. Illumination with short and ultrashort laser pulses produced holes underneath these particles. Calculations of the field near the particles make clear that geometrical optics, that is, focusing by a spherical lens, as well as near-field effects, contribute to the size and shape of these holes. This technique can be utilized for the parallel structuring of large surface areas with a single laser shot.

\section{Introduction}

As industry demands smaller and smaller structures, the production of micro- and nanostructures has attracted much attention in the last decade and still is a progressing field. As a consequence, a lot of different structuring techniques have been developed, pushed by the overwhelming facilities of the scanning probe microscopy. An uncommon approach for the structuring was the utilization of laser radiation by overcoming the diffraction limit. It was proposed that local field enhancement by factors up to 1000 , as can be found in the vicinity of sharp tips (Denk \& Pohl, 1991; Martin \& Girard, 1997; Demming et al., 1998), could result in such a structuring. Indeed, the experiments showed that by illuminating the tip of a scanning tunnelling microscope (STM) by a short laser pulse, nanostructures with lateral dimensions well below $30 \mathrm{~nm}$ can be written on a surface (Gorbunov \& Pompe, 1994; Dickmann \& Jersch, 1995). Further investigations made it clear, however, that

Correspondence. H.-J. Münzer. Tel.: + 49 (0)7531 883856; fax: + 49 (0)7531 883127; e-mail: Hans-Joachim.Muenzer@uni-konstanz.de the nanostructures resulted from the mechanical contact between tip and surface, not from field-induced effects such as field evaporation or local plasma formation (Boneberg et al., 1998b). This contact is a direct consequence of the thermal expansion of the tip because of absorption of laser light (Boneberg et al., 1998a). As this thermal expansion of the tip can amount to several nanometres (Huber et al., 1998), this system is not very suitable for the search for field-induced effects. However, one is not restricted to tip shape geometries but can use other structures as well, provided that they also enhance the field. As such a system we chose transparent submicrometre spherical polystyrene particles to eliminate any thermal effects and focus on the field enhancement.

\section{Mie calculations}

To estimate the field enhancement at the surface in the presence of a dielectric sphere we performed calculations according to the Mie theory (Mie, 1908; Barber \& Hill, 1989). In a first approximation the influence of the surface was neglected, and the calculations were performed for a dielectric sphere (polystyrene; $n=1.58$ ) in vacuum and for a wavelength of $800 \mathrm{~nm}$. This is a reasonable approach for transparent surface materials with a low index of refraction but for different materials the scenario may change (Lukyanchuk et al., 2000).

Figures 1(A) and (B) show the calculated field intensity enhancement for a particle of $1.7 \mu \mathrm{m}$ in diameter. Focusing of the incident radiation at the light averted side generates an enhancement of the field intensity by a factor of 30 . Note that 'field intensity' denotes the square of the electric field vector. This can be understood qualitatively as focusing by a small spherical lens with the concepts of geometrical optics. However, even these particles that are more than double the wavelength in diameter exhibit effects due to near-field 

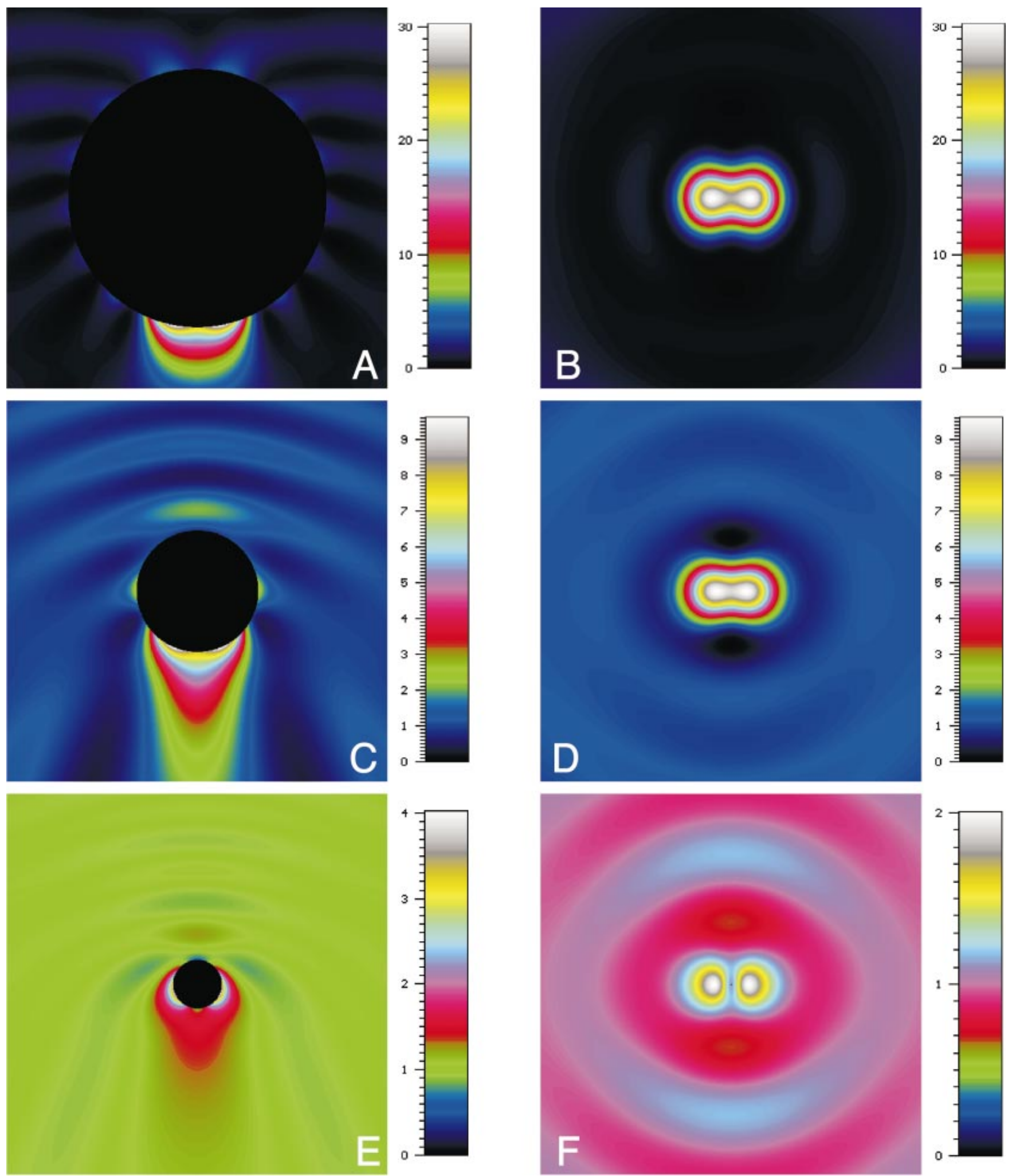

Fig. 1. Calculated intensity enhancement outside of dielectric PS spheres of different diameter in free space for a light wavelength of $800 \mathrm{~nm}$. The intensity in a plane parallel to the propagation and polarization direction is plotted in the left column. The intensity in a plane underneath the particle is shown in the right column. (A) and (B) refer to particles of $1.7 \mu \mathrm{m}$, (C) and (D) of $800 \mathrm{~nm}$, and (E) and (F) of $320 \mathrm{~nm}$ in diameter, respectively. The picture size is $2.5 \mu \mathrm{m} \times 2.5 \mu \mathrm{m}$ for each picture. 
optics and evanescent waves: the incident field is not focused at a distance of

$$
f=r \frac{2-n}{2(n-1)}
$$

from the particle surface as expected from geometrical optics (Bergmann \& Schäfer, 1987), but the maximum field intensity is in this example right at the surface of the particle.

Figures 1(C) and (D) show the same calculations for particles of $800 \mathrm{~nm}$ in diameter. The scenario does not change substantially compared to the $1.7 \mu \mathrm{m}$ particle but the enhancement factor decreases from 30 to 10 , and even though the particles are more than two times smaller the area of high field strength remains nearly the same.

When we compute the field for even smaller spheres, e.g. for particles $320 \mathrm{~nm}$ in diameter, as shown in Figs 1(E) and (F), the regions of maximum field are no longer located on the optical axis opposite to the incident beam, but move sideways along the surface of the sphere. This behaviour originates in the radiation field becoming more and more like that of a dipole as the size of the particle shrinks. The maximum enhancement factor in the lobes is computed to be 4.2 , whereas in the plane underneath the particles it is only about 2 . The pictures in the right column of Fig. 1 show that although the value of the maximum intensity increases from about 2 for the smaller particles to 30 for the larger ones, the field distribution remains nearly the same. The shape resembles an 'infinity sign' $(\infty)$, the distance between the maxima can be determined to be $\approx 300 \mathrm{~nm}$ in all cases.

From these computations it is obvious that underneath the irradiated spheres one deals with light intensities far above the nominal applied ones. This has strong consequences in all techniques where one has to pay attention to threshold processes in laser fluence, e.g. cleaning thresholds in laser-assisted particle removal (Zapka et al., 1991) or ablation thresholds in surface structuring.

\section{Experimental set-up}

\subsection{Sample preparation}

As samples we used industrial silicon wafers with [100] orientation (Wacker Siltronic, Burghausen, Germany) and BK7 glass plates. For cleaning purposes the substrates were sonicated in water and subsequently in isopropyl alcohol (IPA). After this cleaning procedure monodisperse polystyrene (PS) spheres with diameters of $320 \mathrm{~nm}, 800 \mathrm{~nm}$ and $1.7 \mu \mathrm{m}$, respectively, were applied to the samples by spin coating. This technique is described in detail in Mosbacher et al. (1999). The surface of the samples prior and after laser treatment was characterized by a scanning electron microscope (SEM).

\subsection{Laser systems}

To illuminate the samples we used two different laser systems. For some experiments a pulsed Ti:sapphire-laser provided pulses of $100 \mathrm{fs}$ duration with energies up to $10 \mathrm{~mJ}$ pulse $^{-1}$; for the others a pulsed Nd:YAG-laser followed by an optical parametrical oscillator (OPO) supplied nanosecond pulses. The femtosecond pulses were generated by a mode-locked Ti:Sa oscillator (Tsunami, Spectra Physics) and amplified by a Ti:Sa amplifier (TSA, Spectra Physics) with $10 \mathrm{~Hz}$ repetition rate. The energy of the laser pulses was adjusted using a set of grey filters and measured with a pyroelectric power meter. The wavelength was fixed to $800 \mathrm{~nm}$. The nanosecond pulses were generated by a frequency tripled Nd:YAG-laser $(\lambda=355 \mathrm{~nm})$ followed by an OPO, which provides a wavelength range $750-2000 \mathrm{~nm}$ and a pulse duration of $8 \mathrm{~ns}$. In the experiments described here a wavelength of $800 \mathrm{~nm}$ was used for a better comparison with the results to the femtosecond pulses. The laser beams were focused by a lens $(f=150 \mathrm{~mm})$ onto the sample. Therefore, we could also vary the energy density easily by adjusting the distance from the lens to the sample. The laser fluences were determined by imaging the beam profile onto a CCD camera and detecting the total pulse energy for each pulse. Combining the results provides a spatially resolved mapping of the laser fluences in the irradiated region. The irradiated area of the sample was imaged directly using an optical microscope and could be correlated to the map of the laser fluences afterwards. All experiments were performed under ambient conditions.

\section{Results and discussion}

To examine the feasibility of the process and the applicability of the calculations we systematically investigated the formation of holes underneath irradiated particles. After irradiation of $1.7 \mu \mathrm{m}$ particles at normal incidence holes could be found directly below the centre of the particles (Fig. 2(A)). The laser fluence was adjusted to roughly two times the threshold for hole formation, which is about

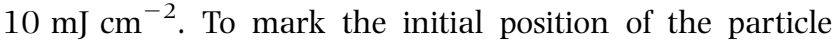
we evaporated a thin $(10 \mathrm{~nm})$ layer of $\mathrm{SiO}_{2}$ onto the sample. (A comparison of the shape of holes formed at samples coated and uncoated by $\mathrm{SiO}_{2}$ showed that the influence of this layer to the optical properties could be neglected.) The typical hole diameter was determined to be $400 \mathrm{~nm}$ and the depth several $10 \mathrm{~nm}$. This is consistent with the calculations in section 2 and can be understood by a focusing effect due to micro-lenses. This became more obvious when we illuminated the spheres at an incident angle of $45^{\circ}$.

Now the hole is no longer located in the centre of the circular area that marks the former position of the colloid, but at its rim, as shown in Fig. 2(B).

Figure 2(C), where the laser beam was also incident at 

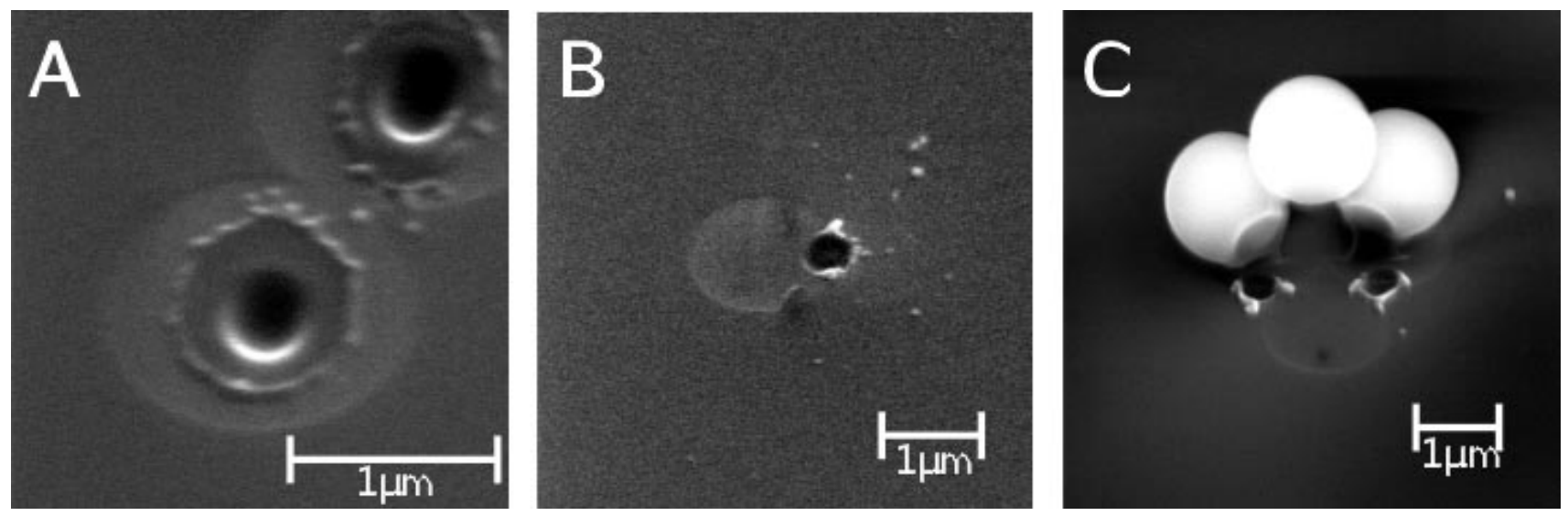

Fig. 2. Holes created by irradiation of $1.7 \mu \mathrm{m}$ particles with a femtosecond laser pulse. (A) normal incidence, (B) $45^{\circ}$ incidence from the left side, (C) $45^{\circ}$ incidence from the back.

$45^{\circ}$, shows that the particles are not removed but 'glued' to the surface by the polymer softened due to the heated silicon substrate. As can be seen, the cluster consisting of three colloid spheres is twisted, probably by the jet of vapour ejected from the holes. However, the foremost particle has not created a real hole, only a change in the 'colour' of the surface is visible. This can be attributed to the shadowing of this particle by the two particles behind.

In this case of $45^{\circ}$ incidence the vapour jet mentioned above is located below the particle boundary, therefore, the particles are accelerated asymmetrically, which results in the twisting of the cluster shown here. At normal incidence, however, if holes are formed the particles are ejected from the surface, which can be attributed to the momentum of the vapour acting directly below the region where the particle adheres to the surface. Previous investigations of Mosbacher et al. (2001) indicated that this is the dominating cleaning mechanism if the particles are removed by femtosecond pulses. By contrast, after illumination with nanosecond pulses the particles are mainly removed by the acceleration of the substrate surface due to thermal expansion (Dobler et al., 1999). Based on this mechanism a process called 'laser cleaning' (Zapka et al., 1991) was developed for the removal of unwanted submicrometre particles from surfaces. However, the formation of substrate damage due to focusing effects limits the maximum laser fluence that can be applied in the laser cleaning process (Leiderer et al., 2000).

To investigate the influence of the duration of the laser pulse to the structuring process we illuminated PS particles of various diameters with nanosecond and femtosecond laser pulses. The results for $800 \mathrm{~nm}$ particles are shown in Fig. 3. The femtosecond laser pulses produced holes with steep walls and a pronounced border, similar to the ones formed under the $1.7 \mu \mathrm{m}$ particles. The laser fluence was adjusted slightly above the threshold for hole formation which is $25 \mathrm{~mJ} \mathrm{~cm}^{-2}$ in this case of $100 \mathrm{fs}$ pulses.
In contrast to these structures the ones formed after illumination with a nanosecond laser pulse are larger and flat, rather than being deep holes. They are surrounded by a wall and a small hillock is located in the centre.

We can explain this behaviour by the heat flow during the relevant time scales. An ultrashort laser pulse excites the electron system of the target on a femtosecond time scale while the energy transfer to the phonon system, i.e. the heating of the lattice takes place on a time scale of a few picoseconds (von der Linde et al., 1997), whereas a nanosecond laser pulse heats the surface as long as the duration of the laser pulse. The thermal diffusion length is given as

$$
l=2 \sqrt{ } D \tau
$$

where $D$ is the thermal diffusion coefficient and $\tau$ is the laser-beam dwell time. This definition is derived by calculating the 1/e decay in the temperature distribution for a point source in infinite space after the time $\tau$. In the case mentioned here (semi-infinite half-space, extensive laser beam, etc.) the situation is more complicated (Bäuerle, 2000), however, only the prefactor of Eq. (2) may change; the order of magnitude remains the same. For a silicon surface we estimate for $\tau=8 \mathrm{~ns}$ a length $l \approx 1 \mu \mathrm{m}$, whereas in the case of a femtosecond pulse the heat diffusion can be neglected on the electron-phonon thermalization timescale. Therefore, in the case of ultrashort laser pulses the surface is heated very fast in a small region. This leads to high temperatures and consequently to explosive evaporation and the formation of small holes with a distinct boundary. In addition, effects like plasma formation, multiphoton absorption and optical breakdown (Bäuerle, 2000) have to be taken into account. In the case of nanosecond laser pulses the heat can diffuse during the duration of the laser pulse and therefore a larger area is heated (and, consequently, the temperatures reached are lower), which 



Fig. 3. Holes created by irradiation of $800 \mathrm{~nm}$ PS spheres with nanosecond and femtosecond laser pulses $(\lambda=800 \mathrm{~nm})$. (A) $100 \mathrm{fs}$, (B) $8 \mathrm{~ns}$.

leads to melting rather than vaporization, thus to the formation of melting pools.

To study the structuring process with spheres smaller than $\lambda / 2$ we illuminated $320 \mathrm{~nm}$ particles with a femtosecond laser pulse. It was still possible to produce holes (Fig. 4(A)), and the threshold fluence for the

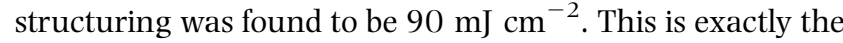
fluence that was applied to the region displayed in Fig. 4(A).

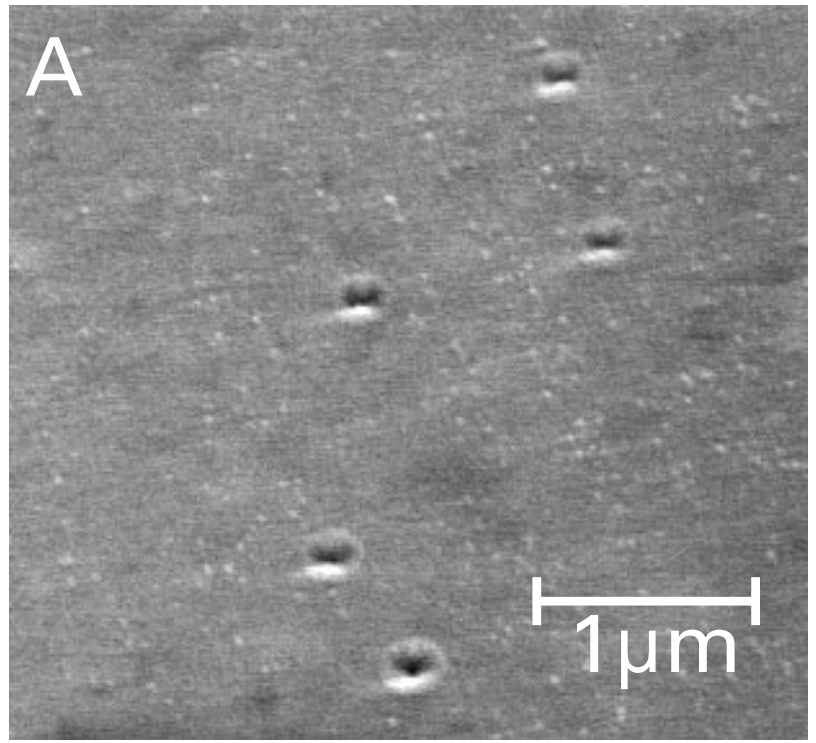

Whereas the hole formation underneath the larger particle sizes could be explained basically in terms of geometrical optics, this is not the case for these smaller particles. The shape of the holes is now indeed as calculated in section 2, as they show a double peak structure, in agreement with the near-field distribution close to the sphere. The structure of the holes in Fig. 4(A) begins to blur from the top to bottom of the picture. This is due to the increase of the laser



Fig. 4. Holes created by irradiation with a femtosecond laser pulse. (A) $320 \mathrm{~nm}$ spheres on Si, (B) $800 \mathrm{~nm}$ spheres on glass. 
fluence. An even further increase in laser fluence results in a complete smearing out of the double-peak structures. Also remarkable is the distance between the two peaks. It is about $100 \mathrm{~nm}$, thus three times smaller than calculated. We attribute this discrepancy to the high index of refraction $(n=3.69, k=0.0065$; Palik, 1985) of the silicon substrate, which influences the field distribution also above the surface.

Figure 4(B) shows the surface modifications after irradiating $800 \mathrm{~nm}$ PS particles on top of a glass surface with a femtosecond pulse. Again, double hole structures form but the distance between the maxima (about $200 \mathrm{~nm}$ ) is larger than before but still smaller than calculated. This can be attributed to the lower index of refraction of glass ( $n=1.51, k=0$, Schott Glas, Mainz, Germany) as well as to the larger particle diameter. Therefore, this technique, as well as being a method for nanostructuring, proves to be suitable for probing the distribution of the near field of small particles. In a recent publication, Kawata et al. (1999) used a polymeric substrate for the same purpose. However, their experiments employed cw lasers and they were not able to image the detailed structure of the field distribution.

\section{Conclusions}

We have shown that it is possible to utilize the near field of small particles to produce holes on silicon and glass surfaces. Although the calculations of the field in the vicinity of these particles were performed in free space, that is the influence of the surface was completely neglected, we could nevertheless interpret the shape of the holes by taking the heat diffusion into account. We could show that it is possible to infer the near field due to the particles close to the surface from the shape of these holes. For small particles and high refractive index surfaces we find deviations from the behaviour predicted by the free space computations. For further investigation, more precise calculations, which take the influence of the substrate into account, have to be carried out. Additionally, a systematic variation of the optical properties of the substrate will provide a deeper insight into the influence of the substrate on the near-field distribution around the particle. As colloidal particles can be applied onto various materials including polymeric, biological and semiconducting ones the technique seems to be feasible for nanostructuring of such technologically promising substrates and will be investigated further. By controlled application of the colloidal suspension we are able to deposit isolated PS spheres at any desired concentration onto the substrate. Spheres, deposited onto a sample in such a way, can be used to create single holes. Another possible method leading to larger hole arrays rather than individual holes is the employment of a $2 \mathrm{D}$ colloidal monolayer as focusing mask (Burmeister et al., 1998). This parallel process would allow the structuring of large substrate areas in a single shot process resulting, e.g. in 1 million holes for a single shot.

\section{Acknowledgements}

This work was supported by the Konstanz Center of Modern Optics and the EU (TMR ERB-CT98-0188 'Modelling and diagnostic of pulsed laser-solid interactions: applications to laser cleaning').

\section{References}

Barber, P.W. \& Hill, S.C. (1989) Light Scattering by Particles: Computational Methods. World Scientific Publishing, Singapore.

Bäuerle, D. (2000) Laser Processing and Chemistry. 3th edn, Springer-Verlag, Berlin.

Bergmann, L. \& Schäfer, C. (1987) Optik, Vol. III, 8th edn. De Gruyter, Berlin.

Boneberg, J., Münzer, H.-J., Tresp, M., Ochmann, M. \& Leiderer, P. (1998a) The mechanism of nanostructuring upon nanosecond laser irradiation of a STM-tip. Appl. Phys. A, 67, 381-384.

Boneberg, J., Tresp, M., Ochmann, M., Münzer, H.-J. \& Leiderer, P. (1998b) Time-resolved measurements of the response of a STMtip upon illumination with a nanosecond pulse. Appl. Phys. A, 66, 615-619.

Burmeister, F., Schäfle, C., Keilhofer, B., Bechinger, C., Boneberg, J. \& Leiderer, P. (1998) From mesoscopic to nanoscopic surface structures: lithography with colloid monolayers. Adv. Mater. 10, $495-497$.

Demming, F., Jersch, J., Dickmann, K. \& Geshev, P.I. (1998) Calculation of the field enhancement on laser-illuminated scanning probe tips by the boundary element method. Appl. Phys. B, 66, 593-598.

Denk, W. \& Pohl, D.W. (1991) Near-field optics: microscopy with nanometer-size fields. J. Vac. Sci. Technol. B, 9, 510-513.

Dickmann, K. \& Jersch, J. (1995) Nanostructuring with laser radiation by field enhancement in the nearfield of a STM tip. Laser Optoelektronik, 27, 76-83.

Dobler, V., Oltra, R., Boquillon, J.P., Mosbacher, M., Boneberg, J. \& Leiderer, P. (1999) Surface acceleration during dry laser cleaning of silicon. Appl. Phys. A, 69, 335-337.

Gorbunov, A.A. \& Pompe, W. (1994) Thin film nanoprocessing by laser/STM combination. Phys. Stat. Sol. (A), 145, 333-338.

Huber, R., Koch, M. \& Feldmann, J. (1998) Laser-induced thermal expansion of a scanning tunneling microscope tip measured with an atomic force microscope cantilever. Appl. Phys. Lett. 7 , 2521-2523.

Kawata, Y., Egami, C., Nakamura, O., Sugihara, O., Okamoto, N., Tsuchimori, M. \& Watanabe, O. (1999) Non-optically probing near-field microscopy. Opt. Commun. 16, 6-12.

Leiderer, P., Boneberg, J., Dobler, V., Mosbacher, M., Münzer, H.-J., Chaoui, N., Siegel, J., Solis, J., Afonso, C.N., Fourrier, T., Schrems, G. \& Bäuerle, D. (2000) Laser-induced particle removal from silicon wafers. Proc. SPIE, 4065, 249-259.

von der Linde, D., Sokolowski-Tinten, K. \& Bialkowski, J. (1997) Laser-solid interaction in the femtosecond time regime. Appl. Surf. Sci. 109/110, 1-10. 
Lukyanchuk, B.S., Zheng, Y.W. \& Lu, Y.F. (2000) Laser cleaning of solid surface: optical resonance and near-field effects. Proc. SPIE, 4065, 576-587.

Martin, O.J.F. \& Girard, Ch. (1997) Controlling and tuning strong optical field gradients at a local probe microscope tip apex. Appl. Phys. Lett. 70, 705-707.

Mie, G. (1908) Beiträge zur Optik trüber Medien, speziell kolloidaler Metallösungen. Annalen Physik, Vierte Folge, Band, 25, 377-445.

Mosbacher, M., Chaoui, N., Siegel, J., Dobler, V., Solis, J., Boneberg,
J., Afonso, C.N. \& Leiderer, P. (1999) A comparison of dry and steam laser cleaning of Si wafers. Appl. Phys. A, 69, 331-334. Mosbacher, M., Münzer, H.-J., Zimmermann, J., Solis, J., Boneberg, J. \& Leiderer, P. (2001) Optical field enhancement effects in laserassisted particle removal. Appl. Phys. A. 72, 41-44.

Palik, E.D. (1985) Handbook of Optical Constants of Solids. Academic Press, New York.

Zapka, W., Ziemlich, W. \& Tam, A.C. (1991) Efficient pulsed laser removal of $0.2 \mu \mathrm{m}$ sized particles from a solid surface. Appl. Phys. Lett. 58, 2217-2219. 